\title{
EXTENDED ELLIPTIC MILD SLOPE EQUATION INCORPORATING THE NONLINEAR SHOALING EFFECT
}

\author{
XIAO Qian-lu ${ }^{\text {a,b }}$ \\ LI Chun-hui a \\ FU Xiao-yan ${ }^{\text {a, }}$ \\ WANG Mei-ju a, \\ a) Key Laboratory of Coastal Disaster and Defence, Ministry of Education, Hohai University, \\ Nanjing, China;

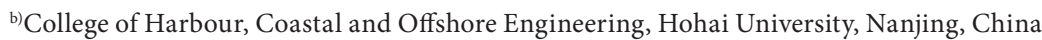

\begin{abstract}
The transformation during wave propagation is significantly important for the calculations of hydraulic and coastal engineering, as well as the sediment transport. The exact wave height deformation calculation on the coasts is essential to near-shore hydrodynamics research and the structure design of coastal engineering. According to the wave shoaling results gained from the elliptical cosine wave theory, the nonlinear wave dispersion relation is adopted to develop the expression of the corresponding nonlinear wave shoaling coefficient. Based on the extended elliptic mild slope equation, an efficient wave numerical model is presented in this paper for predicting wave deformation across the complex topography and the surf zone, incorporating the nonlinear wave dispersion relation, the nonlinear wave shoaling coefficient and other energy dissipation factors. Especially, the phenomenon of wave recovery and second breaking could be shown by the present model. The classical Berkhoff single elliptic topography wave tests, the sinusoidal varying topography experiment, and complex composite slopes wave flume experiments are applied to verify the accuracy of the calculation of wave heights. Compared with experimental data, good agreements are found upon single elliptical topography and one-dimensional beach profiles, including uniform slope and step-type profiles. The results indicate that the newly-developed nonlinear wave shoaling coefficient improves the calculated accuracy of wave transformation in the surf zone efficiently, and the wave breaking is the key factor affecting the wave characteristics and need to be considered in the nearshore wave simulations.
\end{abstract}

Keywords: nonlinear dispersion relation; nonlinear shoaling coefficient; extended elliptic mild slope equation; surf zone; wave transformation

\section{INTRODUCTION}

Waves propagate from deep waters to shallow waters with wave heights changing due to shoaling, refraction, diffraction, reflection, bottom friction and breaking. The transformation above during wave propagation is significantly important for the calculations of hydraulic and coastal engineering, as well as the sediment transport. The breaking waves not only produce large forces on coastal structures but also give rise to near-shore currents which influence the beach topographies [5][10][15]. The exact wave height deformation calculation on the coasts is essential to near-shore hydrodynamics research and the structure design of coastal engineering.

Wave numerical models based on mild slope equation involving breaking have been proposed by many scholars[3] [6][8][11][16-17][19][21-23] and the commonly used method is a wave energy dissipation coefficient for wave breaking, which is applicable to arbitrary reflective boundary conditions. Watanabe and Maruyama applied the time dependent mild slope equation to simulate the wave transformation in the surf zone, and found that the wave height was underestimated at the breaking point when applying a linear wave shoaling coefficient. To overcome this shortcoming of the linear mild slope equation, Black and Rosenberg[17] raised a 
semi-empirical formula, but it is difficult to calculate the combined wave transformation on the coasts. Shuto's empirical nonlinear shoaling equations is applied by Tsai[19] to deduce the wave shoaling coefficient and improve the mild slope equation, which produced better wave heights prediction but emerge restrictions when Ursell number is less than 30.

In this paper, the empirical nonlinear shoaling equations proposed by Shuto are utilized to develop the nonlinear wave shoaling coefficient corresponding to the nonlinear wave dispersion relation. The extended elliptic mild slope equation is modified with the nonlinear wave dispersion relation, the corresponding nonlinear wave shoaling coefficient, the wave frictional energy dissipation coefficient and the wave breaking energy loss coefficient to calculate the wave transformation in the complicated topographies and the surf zone. Compared with the linear wave model, the accuracy of the numerical calculations of modified wave model is improved and the computed wave heights under these topographies conform to the experimental results preferably.

\section{WAVE DISPERSION RELATION}

The nonlinear dispersion relation with higher precision could be expressed as[9]

$$
\sigma^{2}=g k\left(1+p \varepsilon^{2}\right) \tanh (k h+q \varepsilon)
$$

Where, $\sigma$ is the frequency of wave, $g$ is the acceleration of gravity, $\mathrm{k}$ is the wave number, $\mathrm{h}$ is the water depth and the parameters $\mathrm{p}, \mathrm{q}$ and $\varepsilon$ could be written as

$$
p=\tanh (k h) \quad q=\left[\frac{k h}{\sinh (k h)}\right]^{2} \quad \varepsilon=\frac{k H}{2}
$$

Where $\mathrm{H}$ is the wave height. With simple transformations and arrangements, another form of eq.(1) could be expression as follow

$$
L=\frac{g T^{2}\left(1+p \varepsilon^{2}\right)}{2 \pi} \tanh (k h+q \varepsilon)
$$

Where $\mathrm{L}$ is the wave length and $\mathrm{T}$ is the wave period. According to the formula structure of eq. (3), when the ratio of water depth and wave length is larger than 0.5 or the wave belongs to the deep water wave, eq. (3) could be written as

$$
L_{0}=\frac{g T^{2}\left(1+\varepsilon_{0}^{2}\right)}{2 \pi}
$$

Where, $\mathrm{L} 0$ is the wave length in deep water and $\varepsilon_{0}=\mathrm{k}_{0} \mathrm{H}_{0} / 2$. $\mathrm{k}_{0}$ and $\mathrm{H}_{0}$ are the wave number and the wave height in deep water, respectively. Combining eq. (3) and eq. (4), namely

$$
L=L_{0} \frac{1+p \varepsilon^{2}}{1+\varepsilon_{0}^{2}} \tanh (k h+q \varepsilon)
$$

Dividing by the wave period $\mathrm{T}$ at both ends of eq.(5), the relational expression of the wave phase velocity $\mathrm{C}_{\text {and }} \mathrm{C}_{0}$ in deep water could be indicated as

$$
\frac{C}{C_{0}}=\frac{1+p \varepsilon^{2}}{1+\varepsilon_{0}^{2}} \tanh (k h+q \varepsilon)
$$

On the basis of the definition of wave group velocity, the expression of wave group velocity would be written as

$$
C_{g}=\mathrm{d} \sigma / \mathrm{d} k
$$

Taking the derivative of the wave number for both ends of eq. (1), and substituting into eq. (7), the wave group velocity could be expressed as

$C_{g}=\frac{C}{2}+\frac{2 p \varepsilon^{2}+k h \varepsilon^{2} / \cosh ^{2}(k h)}{1+p \varepsilon^{2}} \frac{C}{2}+\frac{C}{\sinh (2 k h+2 q \varepsilon)}\left[k h+q \varepsilon+\frac{2 \varepsilon k^{2} h^{2}}{\sinh ^{3}(k h)}(\sinh (k h)\right.$

$-k h \cosh (k h))]$

Adopting eq. (2) to simplify eq. (8) further, namely

$$
C_{g}=\frac{C}{2}+\frac{2 p \varepsilon^{2}+h k \varepsilon^{2}\left(1-p^{2}\right)}{1+p \varepsilon^{2}} \frac{C}{2}+\frac{C}{2 \sinh (2 k h+2 q \varepsilon)}\left[k h+\varepsilon q\left(3-\frac{2 k h}{p}\right)\right]
$$

Then, the relational expression between wave group velocity $\mathrm{C}_{\mathrm{g}}$ and the wave phase velocity $\mathrm{C}$ could be shown as

$$
C_{g}=C N
$$

Where, the ratio $\mathrm{N}$ could be written as

$N=\frac{1}{2}+\frac{p \varepsilon^{2}+h k \varepsilon^{2}\left(1-p^{2}\right) / 2}{1+p \varepsilon^{2}}+\frac{1}{2 \sinh (2 k h+2 q \varepsilon)}\left[k h+\varepsilon q\left(3-\frac{2 k h}{p}\right)\right]$

When the wave locates in the deep water, eq. (11) could be simplified as

$$
N=\frac{1}{2}+\frac{\varepsilon_{0}^{2}}{1+\varepsilon_{0}^{2}}
$$

\section{WAVE ENERGY EQUATION}

\section{WAVE SHOALING EFFECT}

Wave energy equation is the foundation of wave transformation calculation[4]. In the process of monochromatic wave propagation, one dimension steady energy equation could be given by [1]

$$
\frac{\mathrm{d}}{\mathrm{d} x}\left(E C_{g}\right)=-N f E
$$


Where, $\mathrm{E}$ is the wave energy per unit area of the water body, $\mathrm{f}$ is the coefficient of combined energy dissipation, and $\mathrm{x}$ is the horizontal axis of the Cartesian coordinate perpendicular to the coastline. The wave energy dissipation on the righthand side of eq. (13) could be equal to zero if the energy was assumed without any loss, such as outside the surf zone[19]. According to the Airy wave theory, $\mathrm{E}=\rho \mathrm{gH}^{2} /$ and the wave shoaling coefficient could be shown as

$$
f_{s}=\frac{H}{H_{0}}=\sqrt{\frac{C_{0}}{2 n C}}
$$

Where, $\mathrm{f}_{\mathrm{s}}$ is the wave shoaling coefficient and $\mathrm{n}$ is the ratio of wave group velocity and the wave phase velocity due to the linear dispersion relation. If applying the nonlinear dispersion relation eq. (1), combining eq. (1) with eq. (13), yields

$$
\frac{1}{8} \rho g H_{0}^{2} C_{0}\left(\frac{1}{2}+\frac{\varepsilon_{0}^{2}}{1+\varepsilon_{0}^{2}}\right)=\frac{1}{8} \rho g H^{2} C N
$$

After further arrangement, namely

$$
f_{s}=\frac{H}{H_{0}}=\sqrt{C_{0}\left(\frac{1}{2}+\frac{\varepsilon_{0}^{2}}{1+\varepsilon_{0}^{2}}\right) / C N}
$$

Substituting eq. (11) into eq. (16), the wave shoaling coefficient in the linear wave shoaling theory with nonlinear dispersion relation could be expressed as

$f_{s}=\sqrt{C_{0}\left(\frac{1}{2}+\frac{\varepsilon_{0}^{2}}{1+\varepsilon_{0}^{2}}\right) / C N}=\sqrt{\left(\frac{1}{2}+\frac{\varepsilon_{0}^{2}}{1+\varepsilon_{0}^{2}}\right) \frac{1+\varepsilon_{0}^{2}}{N\left(1+p \varepsilon^{2}\right) \tanh (k h+q \varepsilon)}}$

Thus the liner wave shoaling conclusion proposed by Shuto can be evolved as

$$
\left\{\begin{array}{cc}
\frac{H}{H_{0}}=\sqrt{\left(\frac{1}{2}+\frac{\varepsilon_{0}^{2}}{1+\varepsilon_{0}^{2}}\right) \frac{1+\varepsilon_{0}^{2}}{N\left(1+p \varepsilon^{2}\right) \tanh (k h+q \varepsilon)}} & U_{r} \leq 30 \\
H h^{\frac{2}{7}}=\text { const. } & 30<U_{r} \leq 50 \\
H h^{\frac{5}{2}}\left(\sqrt{U_{r}}-2 \sqrt{3}\right)=\text { const. } & U_{r}>50
\end{array}\right.
$$

Based on the relational expressions as follow

$\frac{\mathrm{d}}{\mathrm{d} x}\left(E C_{g}\right)=\frac{\mathrm{d}}{\mathrm{d} h}\left(E C_{g}\right) \frac{\mathrm{d} h}{\mathrm{~d} x}=-\frac{\mathrm{d}}{\mathrm{d} h}\left(E C_{g}\right) \tan \beta_{p o}=-f_{s} k\left(E C_{g}\right)$

Combining eq. (10), eq. (13) and eq. (19), the expression of wave shoaling coefficient could be shown as

$$
f_{s}=\left(\frac{\mathrm{dC}}{\mathrm{d} h}+\frac{C}{N} \frac{\mathrm{d} N}{\mathrm{~d} h}+\frac{C}{E} \frac{\mathrm{dE}}{\mathrm{d} h}\right) \tan \beta_{p o}
$$

Where, $\beta$ is the bottom slope along the wave transformation direction. Namely, the solution of eq. (20) is the derivation of three differential terms expressed by the wave characteristics.

In eq. (20), the derivative of wave velocity $C$ with respect to the water depth $\mathrm{h}$ could be shown as

$$
\frac{\mathrm{dC}}{\mathrm{d} h}=\frac{\mathrm{d}}{\mathrm{d} h}\left(\frac{\sigma h}{k h}\right)=\frac{\sigma k h-\sigma h \frac{\mathrm{d}(k h)}{\mathrm{d} h}}{(k h)^{2}}
$$

Due to the nonlinear dispersion relation equation (1), yields $\sigma^{2}=g\left(1+p \varepsilon^{2}\right) \tanh (k h+q \varepsilon) \frac{\partial(k h)}{\partial h}+g k h \tanh (k h+q \varepsilon) \frac{\partial\left(1+p \varepsilon^{2}\right)}{\partial h}+g k h(1+$ $\left.+p \varepsilon^{2}\right) \frac{\partial[\tanh (k h+q \varepsilon)]}{\partial h}$

Expanding and arranging the derivatives of composite functions in eq. (22), namely

$\frac{\partial(k h)}{\partial h}+\frac{1}{1+p \varepsilon^{2}}\left(2 p \varepsilon^{2}+\frac{k h \varepsilon^{2}}{\cosh ^{2}(k h)}\right) \frac{\partial(k h)}{\partial h}+\frac{2}{\sinh (2 k h+2 q \varepsilon)}\left[k h+q \varepsilon+\frac{2 \varepsilon(k h)^{2}}{\sinh ^{3}(k h)}\right] \frac{\partial(k h)}{\partial h}=$ $k+\frac{2 k p \varepsilon^{2}}{1+p \varepsilon^{2}}+\frac{2 k q \varepsilon}{\sinh (2 k h+2 q \varepsilon)}$

Combining eq. (11) and eq. (23), the derivative of kh could be written as

$$
\frac{\mathrm{d}(k h)}{\mathrm{d} h}=\frac{k}{2 N}+\frac{k p \varepsilon^{2}}{\left(1+p \varepsilon^{2}\right) N}+\frac{k q \varepsilon}{N \sinh (2 k h+2 q \varepsilon)}
$$

Substituting eq. (24) into eq. (21), yields

$$
\frac{\mathrm{dC}}{\mathrm{d} h}=\frac{C}{h}\left[1-\frac{1}{2 N}-\frac{p \varepsilon^{2}}{\left(1+p \varepsilon^{2}\right) N}-\frac{q \varepsilon}{N \sinh (2 k h+2 q \varepsilon)}\right]
$$

In eq. (20), the derivative of ratio $\mathrm{N}$ with respect to the water depth h could be shown as

$$
\begin{aligned}
& \frac{\mathrm{d} N}{\mathrm{~d} h}=\frac{1}{2\left(1+p \varepsilon^{2}\right)^{2}}\left[\left(1+p \varepsilon^{2}\right) \frac{\partial}{\partial h}\left(2 p \varepsilon^{2}+\frac{k h \varepsilon^{2}}{\cosh ^{2}(k h)}\right)-\left(2 p \varepsilon^{2}+\frac{k h \varepsilon^{2}}{\cosh ^{2}(k h)}\right) \frac{\partial}{\partial h}(1+\right. \\
& \left.\left.p \varepsilon^{2}\right)\right]+\frac{1}{\sinh ^{2}(2 k h+2 q \varepsilon)}\left[\sinh (2 k h+2 q \varepsilon) \frac{\partial}{\partial h}\left(k h+q \varepsilon+\frac{2 \varepsilon k^{2} h^{2}}{\sinh ^{3}(k h)}(\sinh (k h)-k h \cosh (k h))\right)-\right. \\
& \left.\left(k h+q \varepsilon+\frac{2 \varepsilon k^{2} h^{2}}{\sinh ^{3}(k h)}(\sinh (k h)-k h \cosh (k h))\right) \frac{\partial}{\partial h}(\sinh (2 k h+2 q \varepsilon))\right]
\end{aligned}
$$

Expanding and arranging the derivatives of composite functions in eq. (26), namely

$$
\frac{\mathrm{d} N}{\mathrm{~d} h}=K_{w 1} \frac{\mathrm{d}(k h)}{\mathrm{d} h}+K_{w 2}
$$

Where, the parameter $\mathrm{K}_{\mathrm{w} 1}$ could be written as

$K_{w 1}=\frac{1}{1+p \varepsilon^{2}}\left[\frac{2 p \varepsilon^{2}-p^{3} q \varepsilon}{k h}+\frac{\left(\varepsilon+\varepsilon^{2}\right) p^{2} q}{k^{2} h^{2}}\right]-\frac{\left[2 p \varepsilon^{2}+\varepsilon^{2} p^{2} q /(k h)\right]^{2}}{2 k h\left(1+p \varepsilon^{2}\right)^{2}}+\frac{1}{\sinh (2 k h+2 q \varepsilon)}(1-$
$\left.\frac{14 q \varepsilon}{p}+\frac{9 q \varepsilon}{k h}-2 q \varepsilon k h+\frac{k h}{p^{2}}\right)-\frac{2 \cosh (2 k h+2 q \varepsilon)}{\sinh ^{2}(2 k h+2 q \varepsilon)}\left(1+\frac{3 q \varepsilon}{k h}-\frac{2 q \varepsilon}{p}\right)\left(k h+3 q \varepsilon-\frac{2 k h \varepsilon}{p}\right)$ 
And the parameter $\mathrm{K}_{\mathrm{w} 2}$ could be written as

$K_{w 2}=\frac{-1}{1+p \varepsilon^{2}}\left[\frac{2 p \varepsilon^{2}}{h}+\frac{\varepsilon q p^{2}}{2 k h}\right]+\frac{\left[2 p \varepsilon^{2}+\varepsilon^{2} p^{2} q /(k h)\right] p \varepsilon^{2}}{h\left(1+p \varepsilon^{2}\right)^{2}}-\frac{1}{\sinh (2 k h+2 q \varepsilon)}\left(\frac{q \varepsilon}{k h}+\right.$ $\left.2 q \varepsilon \frac{p-k h}{p}\right)+\frac{2 q \varepsilon \cosh (2 k h+2 q \varepsilon)}{\sinh ^{2}(2 k h+2 q \varepsilon)}\left(k h+q \varepsilon+2 q \varepsilon \frac{p-k h}{p}\right)$

On the basis of eq. (5), the relational expression of the wave numbers in the coasts and in deep waters could be expressed as

$$
k_{0} / k=\frac{1+p \varepsilon^{2}}{1+\varepsilon_{0}^{2}} \tanh (k h+q \varepsilon)
$$

Substituting eq. (30) into eq. (27), the parameters $\mathrm{K}_{\mathrm{w} 1}$ and $\mathrm{K}_{\mathrm{w} 2}$ could be written respectively as

$K_{w 1}=\frac{1}{1+p \varepsilon^{2}}\left[\frac{2 p \varepsilon^{2}-p^{3} q \varepsilon}{k h}+\frac{\left(\varepsilon+\varepsilon^{2}\right) p^{2} q}{k^{2} h^{2}}\right]-\frac{\left[2 p \varepsilon^{2}+\varepsilon^{2} p^{2} q /(k h)\right]^{2}}{2 k h\left(1+p \varepsilon^{2}\right)^{2}}+\left(1-\frac{14 q \varepsilon}{p}+\frac{9 q \varepsilon}{k h}-2 q \varepsilon k h+\right.$ $\left.\frac{k h}{p^{2}}\right) \frac{k_{0}\left(1+\varepsilon_{0}^{2}\right)}{2 k\left(1+p \varepsilon^{2}\right) \sinh ^{2}(k h+q \varepsilon)}-\frac{k_{0}^{2}\left(1+\varepsilon_{0}^{2}\right)^{2}+k^{2}\left(1+p \varepsilon^{2}\right)^{2}}{2 k^{2}\left(1+p \varepsilon^{2}\right)^{2} \sinh ^{2}(k h+q \varepsilon)}\left(1+\frac{3 q \varepsilon}{k h}-\frac{2 q \varepsilon}{p}\right)\left(k h+3 q \varepsilon-\frac{2 k h \varepsilon}{p}\right)$

$K_{w 2}=\frac{-1}{1+p \varepsilon^{2}}\left[\frac{2 p \varepsilon^{2}}{h}+\frac{\varepsilon q p^{2}}{2 k h}\right]+\frac{\left[2 p \varepsilon^{2}+\varepsilon^{2} p^{2} q /(k h)\right] p \varepsilon^{2}}{h\left(1+p \varepsilon^{2}\right)^{2}}-\frac{k_{0}\left(1+\varepsilon_{0}^{2}\right)}{2 k\left(1+p \varepsilon^{2}\right) \sinh ^{2}(k h+q \varepsilon)}\left(\frac{q \varepsilon}{k h}+\right.$ $\left.2 q \varepsilon \frac{p-k h}{p}\right)+\left(k h+q \varepsilon+2 q \varepsilon \frac{p-k h}{p}\right) \frac{q \varepsilon\left[k_{0}^{2}\left(1+\varepsilon_{0}^{2}\right)^{2}+k^{2}\left(1+p \varepsilon^{2}\right)^{2}\right]}{2 k^{2}\left(1+p \varepsilon^{2}\right)^{2} \sinh ^{2}(k h+q \varepsilon)}$

In eq. (20), the derivative of wave energy $\mathrm{E}$ with respect to the water depth could be shown as

$$
\frac{C}{E} \frac{\mathrm{d} E}{\mathrm{~d} h}=\frac{C}{H^{2}} \frac{\mathrm{d}\left(H^{2}\right)}{\mathrm{d} h}
$$

According to eq. (19), the expression of wave height is $H_{0} \sqrt{\frac{1}{2}+\frac{\varepsilon_{0}^{2}}{\left.1+\varepsilon_{0}^{2}\right)} \frac{1+\varepsilon_{0}^{2}}{N\left(1+p \varepsilon^{2}\right) \tanh (k h+q \varepsilon)}}$ when the Ursell number is no larger than 30 , and eq. (33) could be shown as

$$
\frac{C}{E} \frac{\mathrm{d} E}{\mathrm{~d} h}=\frac{C}{H^{2}} \frac{\mathrm{d}\left(H^{2}\right)}{\mathrm{d} h}=-\frac{\mathrm{d} C}{\mathrm{~d} h}-\frac{C}{N} \frac{\mathrm{d} N}{\mathrm{~d} h}
$$

When value range of the Ursell number is $30<\mathrm{U}_{\mathrm{r}} \leq 50$, $\mathrm{H}=\mathrm{C}_{\mathrm{wl}} \mathrm{h}^{-2 / 7}$ and $\mathrm{C}_{\mathrm{w} 1}$ is the coefficient. Namely,

$$
\frac{C}{E} \frac{\mathrm{d} E}{\mathrm{~d} h}=\frac{C}{H^{2}} \frac{\mathrm{d}\left(H^{2}\right)}{\mathrm{d} h}=-\frac{4}{7} \frac{C}{h}
$$

When the Ursell number is larger than $50, H h^{\frac{5}{2}}\left(\sqrt{U_{r}}-2 \sqrt{3}\right)=C_{w 2}$ and $\mathrm{C}_{\mathrm{w} 2}$ is the coefficient. Substituting the expression of the Ursell number, $\mathrm{U}_{\mathrm{r}}=\mathrm{gHT}^{2} / \mathrm{h}^{2}$, into the relational expression of wave height, yields

$$
H h^{\frac{5}{2}}(T \sqrt{g H} / h-2 \sqrt{3})=C_{w 2}
$$

Taking the derivative of the water depth $\mathrm{h}$ for both ends of eq. (36), then $\frac{3}{2} H^{\frac{1}{2}} h^{\frac{3}{2}} \operatorname{Tg}^{\frac{1}{2}} \frac{\mathrm{d} H}{\mathrm{~d} h}+\frac{3}{2} H^{\frac{3}{2}} h^{\frac{1}{2}} \operatorname{Tg}^{\frac{1}{2}}-2 \sqrt{3}\left(h^{\frac{5}{2}} \frac{\mathrm{d} H}{\mathrm{~d} h}+\frac{5}{2} h^{\frac{3}{2}}\right)=0$

After the arrangement, eq. (37) could be transformed as

$$
\frac{\mathrm{d} H}{\mathrm{~d} h}=-\frac{H}{h} \frac{1.5 \sqrt{U_{r}}-5 \sqrt{3}}{1.5 \sqrt{U_{r}}-2 \sqrt{3}}
$$

Substituting eq. (38) into eq. (34), yields

$$
\frac{C}{E} \frac{\mathrm{d} E}{\mathrm{~d} h}=-\frac{C}{h} \frac{3 \sqrt{U_{r}}-10 \sqrt{3}}{1.5 \sqrt{U_{r}}-2 \sqrt{3}}
$$

Combining eq. (25), eq. (27), eq. (34), eq. (35), eq. (39) with eq. (20), the wave nonlinear shoaling coefficient corresponding to the wave nonlinear dispersion relation raised by Li et al.[13] could be expressed as

$$
f_{s}=\left\{\begin{array}{cc}
0 & U_{r} \leq 30 \\
\left(\frac{C}{h}\right)\left[\frac{3}{7}-\frac{K_{w 3}}{k}+\frac{h K_{w 3} K_{w 1}}{N}+\frac{h K_{w 2}}{N}\right] \tan \beta & 30<U_{r} \leq 50 \\
\left(\frac{C}{h}\right)\left[\frac{-4.5 \sqrt{U_{r}}-12 \sqrt{3}}{1.5 \sqrt{U_{r}}-2 \sqrt{3}}+\frac{h\left(K_{w 3} K_{w 1}+K_{w 2}\right)}{N}-\frac{K_{w 3}}{k}\right] \tan \beta & U_{r}>50 \\
\text { (40) }
\end{array}\right.
$$

Where, the parameters $\mathrm{K}_{\mathrm{w} 1}$ and $\mathrm{K}_{\mathrm{w} 2}$ could be expressed as eq. (31) and eq. (32), respectively. The parameter $\mathrm{K}_{\mathrm{w} 3}$ could be written as

$$
K_{w 3}=\frac{k}{2 N}+\frac{k p \varepsilon^{2}}{\left(1+p \varepsilon^{2}\right) N}+\frac{k q \varepsilon}{N \sinh (2 k h+2 q \varepsilon)}
$$

Eq. (40) would be adopted as the wave shoaling coefficient by the extended elliptic mild slope equation in this paper.

\section{WAVE BREAKING EFFECT}

The breaking index considering the bottom slope is following[15]

$$
\begin{gathered}
\xi_{o}=\frac{\tan \beta_{p o}}{\sqrt{H_{0} / L_{0}}} \\
\xi_{b}=\frac{\tan \beta_{p o}}{\sqrt{H_{b} / L_{0}}}
\end{gathered}
$$

$$
\begin{array}{cccc}
\xi_{b} \geq 2.0 & \text { Or } & \xi_{o} \geq 3.3 & \text { surging breaking } \\
0.4 \leq \xi_{b}<2.0 & \text { Or } & 0.5 \leq \xi_{o}<3.3 & \text { plunging breaking } \\
\xi_{b}<0.4 & \text { Or } & \xi_{o}<0.5 & \text { spilling breaking }
\end{array}
$$

Based on the convenience of programming and the distinct classification, the breaking index proposed by Battjes 
would be adopted in the mild slope equation in this paper. The hydraulic jump model is used to compute the energy loss after the wave breaks. Subscribing the index above into wave energy equation, we can get the wave breaking energy dissipation coefficients:

$$
\begin{gathered}
\mathrm{d}\left(E C_{g}\right) / \mathrm{d} x=-0.01875 \rho g\left(H^{2} \sqrt{g h}-0.16 h^{2} \sqrt{g h}\right) / h \\
f_{d}=\frac{0.15}{k h}\left(1-\frac{0.04}{\gamma_{a}^{2}}\right)
\end{gathered}
$$

\section{WAVE FRICTIONAL EFFECT}

In the most coasts, the energy dissipation produced by the bed frictions would be the primary cause compared with those by the permeability loss and the mud surface wave resistance loss.

According to the wave boundary layer theory, the work of the bed friction could be indicated as

$$
D_{f}=f_{b} k\left(E C_{g}\right)
$$

Where, $D_{f}$ is the unit bed surface energy loss and $f_{b}$ is the wave frictional energy dissipation coefficient. And the unit bed surface energy loss could be shown as

$$
D_{f}=\overline{U_{b} \tau_{b}}=\frac{1}{T} \int_{0}^{T} \frac{1}{2} \rho f_{w} U_{b}^{2}\left|U_{b}\right| d t=\frac{1}{2} \rho f_{w} U_{b m}^{3}\left(\frac{1}{T} \int_{0}^{T}\left|\cos ^{3}(\sigma t)\right| d t\right)
$$

Where $\mathrm{U}_{\mathrm{b}}=\mathrm{U}_{\mathrm{bm}} \cos (\mathrm{kx}-\sigma \mathrm{t})=\mathrm{A}_{\mathrm{m}} \sigma \cos (\mathrm{kx}-\sigma \mathrm{t})=(\pi \mathrm{H} /$ $\operatorname{Tsinh}(\mathrm{kh})) \cos (\mathrm{kx}-\sigma \mathrm{t})$. Combing eq. (47) and eq. (48), yields

$$
\begin{gathered}
D_{f}=\frac{2 \pi^{2}}{3} \rho f_{w} \frac{H^{3}}{T^{3} \sinh ^{3}(k h)} \\
f_{b}=\frac{2 f_{w}}{3 \pi} \frac{H \sigma^{2}}{N g \sinh ^{3}(k h)}
\end{gathered}
$$

Eq. (50) would be adopted in this paper for the wave energy dissipation due to the bed frictions.

\section{THE WAVE MODEL BASED ON EXTENDED ELLIPTIC MILD SLOPE EQUATION}

The extended elliptic mild slope equation involving the energy dissipation stated above is applied to calculate the wave transformation. The governing equation of the extended elliptic mild slope equation [11] could be expressed as

$\nabla\left(C C_{g} \nabla \Phi\right)+k^{2} C C_{g}(1+i f) \Phi+\left[f_{1} g \nabla^{2} h+f_{2}(\nabla h)^{2} g k\right] \Phi=0$
Where

$f_{1}=\frac{-4 k h \cosh (k h)+\sinh (3 k h)+\sinh (k h)+8(k h)^{2} \sinh (k h)}{8 \cosh ^{3}(k h)[2 k h+\sinh (2 k h)]}-\frac{k h \tanh (k h)}{2 \cosh ^{2}(k h)}$

$f_{2}=\frac{\operatorname{sech}^{2}(k h)}{6[2 k h+\sinh (2 k h)]^{3}}\left[8(k h)^{4}+16(k h)^{3} \sinh (2 k h)-9 \sinh ^{2}(2 k h) \cosh (2 k h)+\right.$ $\left.12(k h)\left(1+2 \sinh ^{4}(k h)\right)(k h+\sinh (2 k h))\right]$

Where $\nabla=(\partial / \partial \mathrm{x}, \partial / \partial \mathrm{y})$ is the horizontal operator; $\Phi$ is the velocity potential function of the harmonic wave; $\nabla$ hand $\mathrm{V}_{\mathrm{h}}{ }^{2}$ are the bottom slopes and bottom curvatures in the and directions, respectively; and are the two horizontal coordinates; $\mathrm{f}=\mathrm{f}_{\mathrm{s}}+\mathrm{f}_{\mathrm{d}}+\mathrm{f}_{\mathrm{b}}$ is the combined energy dissipation factor, and $f_{s}, f_{b}$ and $f_{d}$ are calculated with eq.(41), eq.(49-1) and eq.(56) respectively. The nonlinear wave dispersion relation is adopted with eq. (1).

In order to verify the present model and test the computational accuracy, the classical and the slope flume experiments would be used including the Berkhoff single elliptical topography, sinusoidal varying topography, and uniform slope and composite slopes topography tests in the flume. In terms of reflecting the modification, the computed values of RIDE model established by Maa et al.[11], which adopted the linear wave theory, would be used to contrast with the present model.

\section{BERKHOFF SINGLE ELLIPTICAL TOPOGRAPHY}

Berkhoff et al.[2] conducted the wave propagating and transforming experiment on the ideal uniform slope topography with a single ellipse, and obtained the measured data of eight cross sections. In the numerical simulation, the height of incident wave is $0.0232 \mathrm{~m}$, the period is $1.0 \mathrm{~s}$ and the direction is along the positive coordinate. The comparisons among computed values of the linear wave model of Maa et al., calculated results of the present model and experimental data in the cross section $1 \# \sim 8 \#$ are shown in Fig.2. The horizontal coordinate is the cross section, and the vertical coordinate is the ratio of local wave height and incident wave height. The black solid points in Fig.2 represent the experimental wave height data, the black dashed lines represent computed values of the linear wave model of Maa et al. and the black solid lines represent the calculated results of the present wave model. On the basis of the contrasts in Fig.2, although there are some obvious deviations in some locations, such as the significant error at $17 \mathrm{~m}$ nearby in the $7 \#$ cross section, yet the calculated results of the present model coincide to the experimental results overall and the computed precision is preferable than the linear wave model of Maa et al.. Fig. 3 shows the contrast about the computed wave height distribution fields between the linear wave model of Maa et al. and the present model. And the left figures are the computed values of the linear wave model of Maa et al., and the right figures are the calculated results of the present model. Based on the comparison of wave height distribution field, the calculated results of the present model make the wave energy more disperse after the single ellipse. 
The calibrations indicate that the nonlinear wave dispersion relation and the corresponding wave shoaling coefficient in the present model are able to improve the wave refraction and diffraction effects apparently under the complex topography.

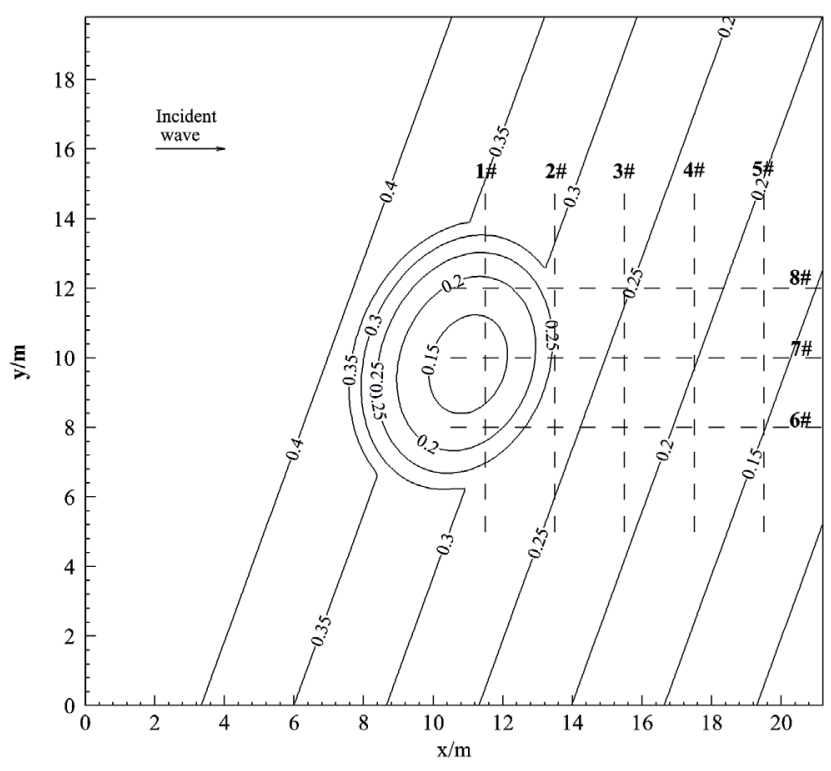

Fig. 1. The single elliptical topography and arrangement of cross sections
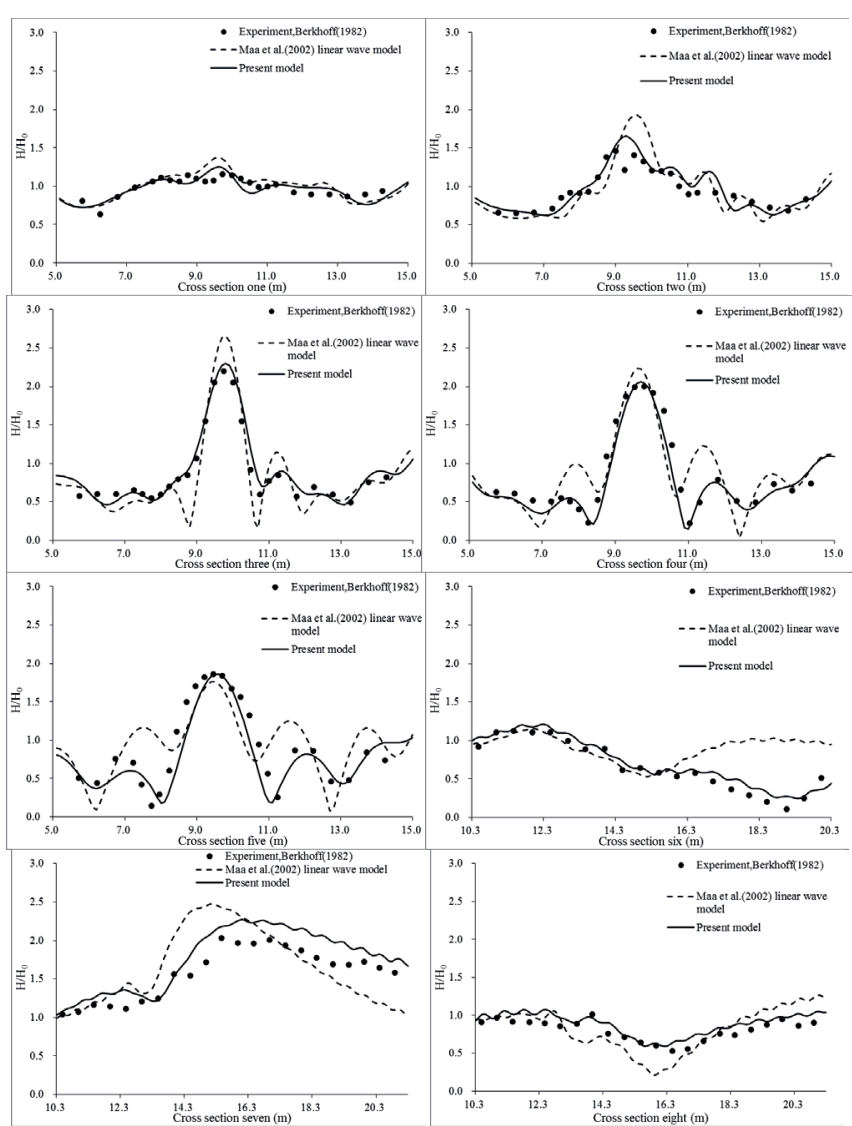

Fig. 2. Compared computed values of the linear wave model of Maa et al.and calculated results of the present model with experimental wave height data in the eight different cross sections
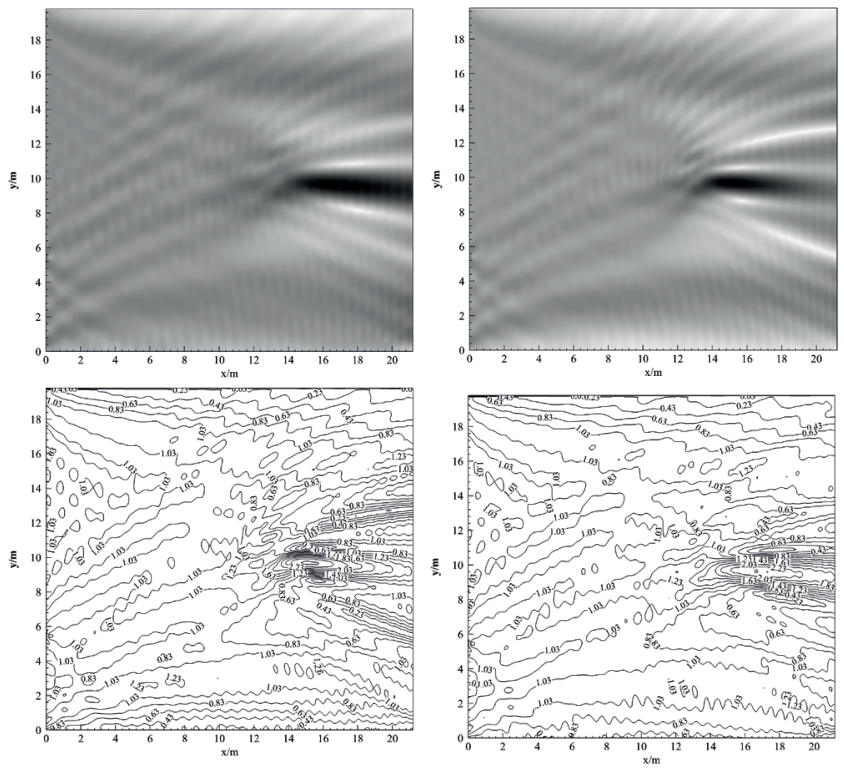

Fig. 3. Compared computed wave height distribution field of the linear wave model of Maa et al. (left)with calculated wave height distribution field of the present model(right)

\section{SINUSOIDAL VARYING TOPOGRAPHY}

Davies and Heathershaw[20] carried out a series of experimental research on the wave transformation under various sand ripple numbers and water depth conditions. The wave situation with ten sand ripples is simulated by the present model. In the numerical simulation, the wave height of incident wave is $0.02 \mathrm{~m}$, the period is $1.31 \mathrm{~s}$, and the direction is along the positive coordinate. The fully absorbing condition is situated at $\mathrm{x}=30 \mathrm{~m}$. The comparisons between observed data and computed values under sinusoidal sand ripples terrain are listed in Fig.5. The horizontal coordinate is the distance from the wave incident position, and the vertical coordinate is the wave amplitude of local wave (Half of local wave height). The black solid points in Fig. 5 represent the observed wave amplitude data, and the black solid lines represent the calculated results of established wave models. In the light of the verifications in Fig.5, the calculated results of the wave mathematical model could coincide to the experimental data generally, and is capable of reflecting the tendency of wave deformation under the sinusoidal varying topography.

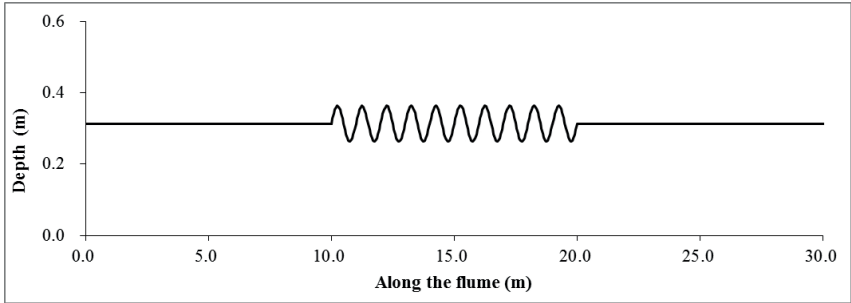

Fig. 4. Sinusoidal varying topography profile when ripple wavenumber equals to ten 


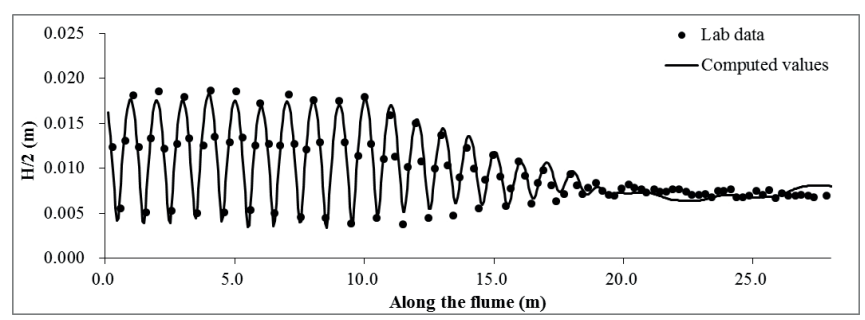

Fig. 5. Compared experimental wave height data observed in the flume with computed values of the present model

\section{COMPOSITE SLOPES TOPOGRAPHY}

The wave transformation experiment under the complex bar-type beach profile caught out by Nagayama[12] is applied to verify the present model, the topography profile is shown in Fig.6(a). The wave height of incident wave is $0.07 \mathrm{~m}$, and the wave period is $1.18 \mathrm{~s}$. The numerical results of the present model are compared with the computed values of the linear wave model of Maa et al. and the experimental data, which are shown in Fig.6(b). Both the solution of the linear wave model of Maa et al. and the present model predict a second wave breaking at the tailing section of the $1 / 20$ slope. For under this bar-type topography, the wave deformation includes the wave shoaling effect, wave frictional effect, wave decaying effect, wave recovery and the second breaking. It is found that the present model is in better agreement with the experimental results and of higher accuracy.

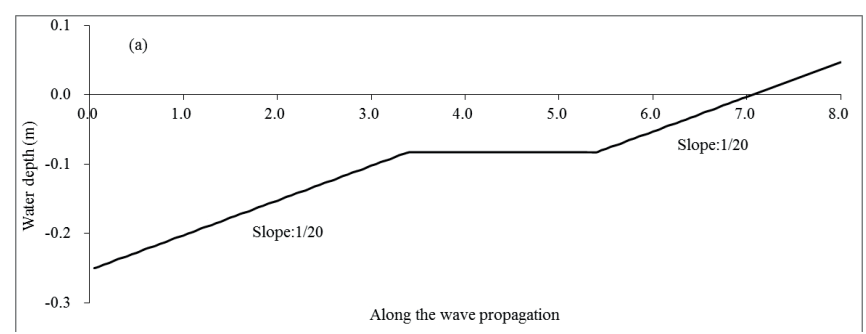

Fig. 6(a). Composite slopes topography profile with fronting and tailing slope $1 / 20$

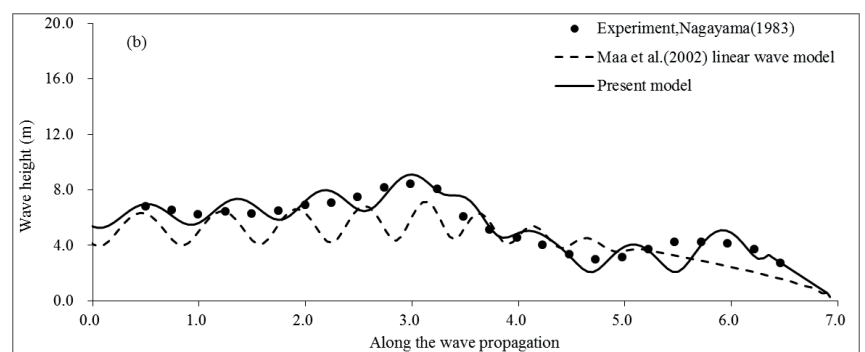

Fig. 6(b). Compared computed values of the linear wave model of Maa et al. and calculated results of the present model with experimental wave height data along the wave propagation

\section{CONCLUSION}

The mild slope equation models applied in this paper have contained various modifications for the computations of combined wave shoaling, refraction, diffraction, reflection, bottom friction and breaking. In the surf zone, linear wave dispersion relation and linear wave shoaling coefficient have been adopted to improve the accuracy of wave height calculation. To overcome the error of the linear dispersion relation, the empirical nonlinear shoaling relation equations are utilized to develop the nonlinear wave shoaling coefficient corresponding to the nonlinear wave dispersion relation. The extended elliptic mild slope equation is modified with the nonlinear wave dispersion relation, the corresponding deduced nonlinear wave shoaling coefficient, the wave frictional energy dissipation coefficient and the wave breaking energy loss coefficient to calculate the wave transformation in the complicated topographies and the surf zone. The Berkhoff classical single elliptic topography experiment, some uniform slope and composite slope flume tests are applied to verify the modified wave model, and the calculated results coincide to the experimental data overall.

\section{ACKNOWLEDGEMENTS}

This work was supported by the Foundation of Jiangsu Province in China ordinary graduate student research innovation project for 2015(Grant No. KYZZ15_0143).

\section{REFERENCES}

1. Akbarpour Jannat, M. R., \& Asano, T. (2007). External forces of sediment transport in surf and swash zones induced by wave groups and their associated long waves. Coastal Engineering Journal, 49(02), 205-227.

2. Berkhoff, J. C. W., Booy, N., \& Radder, A. C. (1982). Verification of numerical wave propagation models for simple harmonic linear water waves. Coastal Engineering, 6(3), 255-279.

3. Cerrato, A., González, J. A., \& Rodríguez-Tembleque, L. (2016). Boundary element formulation of the Mild-Slope equation for harmonic water waves propagating over unidirectional variable bathymetries. Engineering Analysis with Boundary Elements, 62, 22-34.

4. Chang, G., Ruehl, K., Jones, C. A., Roberts, J., \& Chartrand, C. (2016). Numerical modeling of the effects of wave energy converter characteristics on nearshore wave conditions. Renewable Energy, 89, 636-648.

5. Chella, M. A., Bihs, H., Myrhaug, D., \& Muskulus, M. (2015). Hydrodynamic characteristics and geometric properties of plunging and spilling breakers over impermeable slopes. Ocean Modelling.

6. Hamidi, M. E., Hashemi, M. R., Talebbeydokhti, N., \& Neill, S. P. (2012). Numerical modelling of the mild slope equation using localised differential quadrature method. Ocean Engineering, 47, 88-103. 
7. [7] Korotkevich, A. O., Dyachenko, A. I., \& Zakharov, V. E. (2016). Numerical simulation of surface waves instability on a homogeneous grid. Physica D: Nonlinear Phenomena, 321, 51-66.

8. [8] Lin, X., \& Yu, X. (2015). A finite difference method for effective treatment of mild-slope wave equation subject to non-reflecting boundary conditions. Applied Ocean Research, 53, 179-189.

9. Li, R. J., Zhang, Y., \& Gao, H. S. (2004). A wave nonlinear dispersion relation and its application. Ocean Eng./Haiyang Gongcheng, 22(3), 20-24.

10. Lupieri, G., \& Contento, G. (2015). Numerical simulations of 2-D steady and unsteady breaking waves. Ocean Engineering, 106, 298-316.

11. Maa, J. Y., Hsu, T. W., \& Lee, D. Y. (2002). The RIDE model: an enhanced computer program for wave transformation. Ocean Engineering, 29(11), 1441-1458.

12. Nagayama, S. (1983). Study on the change of wave height and energy in the surf zone. Bachelor thesis, Yokohama National University, Japan (in Japanese).

13. Rincon, M. A., \& Quintino, N. P. (2016). Numerical analysis and simulation for a nonlinear wave equation. Journal of Computational and Applied Mathematics, 296, 247-264.

14. Salmon, J. E., \& Holthuijsen, L. H. (2015). Modeling depthinduced wave breaking over complex coastal bathymetries. Coastal Engineering, 105, 21-35.

15. Salmon, J. E., Holthuijsen, L. H., Zijlema, M., van Vledder, G. P., \& Pietrzak, J. D. (2015). Scaling depth-induced wavebreaking in two-dimensional spectral wave models. Ocean Modelling, 87, 30-47.

16. Sharma, A., Panchang, V. G., \& Kaihatu, J. M. (2014). Modeling nonlinear wave-wave interactions with the elliptic mild slope equation. Applied Ocean Research, 48, 114-125.

17. Stockdon, H. F., Holman, R. A., Howd, P. A., \& Sallenger, A. H. (2006). Empirical parameterization of setup, swash, and runup. Coastal engineering, 53(7), 573-588.

18. Thompson, D. A., Karunarathna, H., \& Reeve, D. (2016). Comparison between wave generation methods for numerical simulation of bimodal seas. Water Science and Engineering, 9(1), 3-13.

19. Tsai, C. P., Chen, H. B., Hwung, H. H., \& Huang, M. J. (2005). Examination of empirical formulas for wave shoaling and breaking on steep slopes. Ocean Engineering, 32(3), 469-483.
20. Yu, J., \& Zheng, G. (2012). Exact solutions for wave propagation over a patch of large bottom corrugations. Journal of Fluid Mechanics, 713, 362-375.

21. Zhao, L., Panchang, V., Chen, W., Demirbilek, Z., \& Chhabbra, N. (2001). Simulation of wave breaking effects in two-dimensional elliptic harbor wave models. Coastal Engineering, 42(4), 359-373.

22. Yoon, BI; Woo, SB. (2013). Tidal asymmetry and flood/ ebb dominance around the Yeomha channel in the Han River Estuary, South Korea. Journal of coastal research, $65,1242-1246$.

23. Chen, M; Han, DF. (2015). Multi-grid model for crowd's evacuation in ships based on cellular automata. Polish maritime research, 22(1), 75-81. 